\title{
Finding Bicliques in Digraphs: Application into Viral-Host Protein Interactome
}

\author{
Malay Bhattacharyya ${ }^{1}$, Sanghamitra Bandyopadhyay ${ }^{1}$, and Ujjwal Maulik ${ }^{2}$ \\ 1 Machine Intelligence Unit, Indian Statistical Institute \\ 203 B. T. Road, Kolkata - 700108, India \\ \{malay_r, sanghami\}@isical.ac.in \\ 2 Department of Computer Science and Engineering, Jadavpur University \\ Kolkata - 700032, India \\ umaulik@cse.jdvu.ac.in
}

\begin{abstract}
We provide the first formalization true to the best of our knowledge to the problem of finding bicliques in a directed graph. The problem is addressed employing a two-stage approach based on an existing biclustering algorithm. This novel problem is useful in several biological applications of which we focus only on analyzing the viral-host protein interaction graphs. Strong and significant bicliques of HIV-1 and human proteins are derived using the proposed methodology, which provides insights into some novel regulatory functionalities in case of the acute immunodeficiency syndrome in human.
\end{abstract}

\section{Introduction}

The problem of finding bicliques, i.e. complete subgraphs in an undirected bipartite graph, is a well-known NP-complete problem [4. Bipartite graphs are recognized as a triplet $\left(V_{1}, V_{2}, E\right)$ in general, where $V_{1}, V_{2}$ denote two distinct sets of vertices and $E \subseteq V_{1} \times V_{2}$ is a set of edges. For many real-life applications, directions are also included in graphs depicting regulatory information and they are formalized as a directed graph (digraph) $G$, where we distinguish between the edges $(i, j)$ and $(j, i)$. In several emerging applications, we are interested in exploring biclique-like compact structures in digraphs. In this paper, we formalize the problem of finding bicliques in digraphs. We show that there is a correspondence between the problem of finding bicliques in digraphs and a special type of biclustering. Based on this observation, we propose a method for exploring bicliques in digraphs.

Various studies on gene expression datasets have contributed a lot in the development of different biclustering algorithms. A quality review of majority of the existing biclustering approaches like Cheng and Church's algorithm (CCA) [3], SAMBA [9], co-clustering, etc. can be found in [8] and 7]. Broadly speaking, biclusters are of four types - fixed value, fixed row/column, additive coherent value, and coherent evolution [6. While SAMBA and co-clustering are designed to find constant value biclusters, CCA can simultaneously find constant value and constant row/column biclusters. On the other side, SAMBA is able to uncover overlapping biclusters, while co-clustering (which is designed to only look 
for disjoint patterns) and Cheng and Church's method (which masks the identified bicluster with random values in each iteration) can't find such biclusters. Another divide-and-conquer based biclustering algorithm has recently been proposed that pursues inclusion-maximal procedure to prune out all-0 submatrices recursively from the input binary adjacency matrix to obtain overlapping biclusters $[8$. This exact algorithm is applicable only to binary data and thus more suitable to finding bicliques. As can be seen reviewing the literature, only the heuristic algorithm SAMBA has considered the directional changes in expression values while finding statistically significant biclusters. But unfortunately, none of these methods are applicable to directed graphs where directions are also considered in the graph structure.

In this paper, we revise the methodology proposed in 8 , to find out bicliques in digraphs where all the directions involved in the substructure are monodirectional. We define this as directed bicliques. The problem of finding directed bicliques is formalized in the following section. Sections 4 and 5 describe the proposed methodology and empirical results on the HIV-1-human protein interactome (the comprehensive set of interactions) forming a graph structure, respectively. Finally, Section 5 concludes the paper.

\section{Problem Definition}

A directed bipartite graph, in a generalized form, can be represented as a triplet $G=\left(V_{1}, V_{2}, E\right)$, where $V_{1} \cap V_{2}=\phi$ and $E \subseteq\left\{V_{1} \times V_{2}\right\} \cup\left\{V_{2} \times V_{1}\right\}$. We define a directed biclique, hereafter referred as DBClique, as a complete subgraph of a directed bipartite graph as per the following.

Definition 1 (DBClique). A DBClique is a fully connected subgraph $G^{\prime}=$ $\left(V_{1}^{\prime}, V_{2}^{\prime}, E^{\prime}\right) \subseteq G$ of a directed bipartite graph $G$ such that either $i \in V_{1}^{\prime}, j \in$ $V_{2}^{\prime}, \forall(i, j) \in E^{\prime}$ or $i \in V_{2}^{\prime}, j \in V_{1}^{\prime}, \forall(i, j) \in E^{\prime}$.

A directed bipartite graph is shown in Fig. 1(a) in which the sets of vertices \{II, III $\}$ and $\{2,3,4\}$ form a DBClique. Notably, this corresponds to a special kind of constant value biclusters. If we construct an interaction matrix (as shown in Fig. 1(b)) from the directed bipartite graph in Fig. 1(a), it somehow corresponds to an all-1 submatrix, but not an exact one. We provide a lemma to highlight a correspondence between the problem of finding DBCliques and a variant of biclustering. For this, let us first define the matrix representation of a directed bipartite graph as follows.

Definition 2 (Interaction matrix of a directed bipartite graph). The interaction matrix of a directed bipartite graph $G=\left(V_{1}, V_{2}, E\right)$ is defined as a $\left|V_{1}\right| \times\left|V_{2}\right|$ matrix $\mathcal{I}$ such that

$$
\mathcal{I}_{i j}=\left\{\begin{array}{l}
0, \quad \text { if }(i, j) \notin E \text { and }(j, i) \notin E \\
1, \quad \text { if }(i, j) \in E \text { and }(j, i) \notin E \\
-1, \text { if }(i, j) \notin E \text { and }(j, i) \in E \\
X, \quad \text { if }(i, j) \in E \text { and }(j, i) \in E
\end{array},\right.
$$

$\forall i \in V_{1}, j \in V_{2}$. 


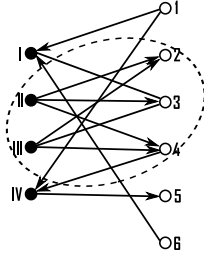

(a)

\begin{tabular}{|c|c|c|c|c|c|}
\hline 1 & 2 & 3 & 4 & 5 & \\
\hline-1 & $\mathbf{0}$ & $X$ & 0 & $\mathbf{0}$ & -1 \\
\hline 0 & 1 & 1 & 1 & 0 & 0 \\
\hline 0 & 1 & $\boldsymbol{X}$ & 1 & 0 & 0 \\
\hline-1 & 0 & 0 & $|-1|$ & \begin{tabular}{|l|l} 
\\
\end{tabular} & 0 \\
\hline
\end{tabular}

(b)

Fig. 1. The one-to-one correspondence between a single (a) DBClique in a directed bipartite graph and (b) a special kind of bicluster in its representative interaction matrix reflected as a submatrix

An example interaction matrix is shown in Fig. 1(b). The character ' $X$ ' in the matrix $\mathcal{I}$ defined beforehand is behaviorally a don't care entry. Based on this representation, we can equate a DBClique to a bicluster through the following lemma.

Lemma 1. Given a directed bipartite graph $G=\left(V_{1}, V_{2}, E\right)$, a DBClique $G^{\prime}=$ $\left(V_{1}^{\prime}, V_{2}^{\prime}, E^{\prime}\right) \subseteq G$ corresponds to a bicluster in the interaction matrix of $G$ such that all the elements in the submatrix are either ' 1 ' or ' -1 ', with the entries of ' $X$ ' additionally allowed.

Proof. Certainly, an entry ' $X$ ' in any submatrix of a bicluster represents both ' 1 ' and ' -1 '. Thus, a bicluster with all the entries either ' 1 ' or ' $X$ ' will correspond to an edge $(i, j)$ directed from $V_{1}$ to $V_{2}$. Equivalently, a bicluster with all '-1' or ' $X$ ' will correspond to an edge $(i, j)$ directed from $V_{2}$ to $V_{1}$. Thus the lemma.

Our motivation is to find out the set of maximal DBCliques from a given directed bipartite graph.

\section{Proposed Method}

We describe the two-stage algorithm to find out DBCliques in digraphs using the previous lemma. The methodology is based on the Bimax algorithm [8]. The approach for finding out maximal overlapping DBCliques (all ' 1 ' and all '-1' biclusters) is described in detail in Algorithm[1. The key step in this algorithm is pursuing separate phases (steps 2-5 and steps 6-9) for finding out the regulating (all ' 1 ') and regulated (all ' -1 ') DBCliques. After this, we apply a divide-andconquer process (adopted in [9]) to find out the maximal DBCliques from the given directed bipartite graph.

\section{Experimental Results}

To demonstrate the effectiveness of the methodology, we collect the interaction information (5134 interactions in total) between 19 HIV-1 and 1448 human proteins as reported in a recently published dataset [5]. It consists of two types 


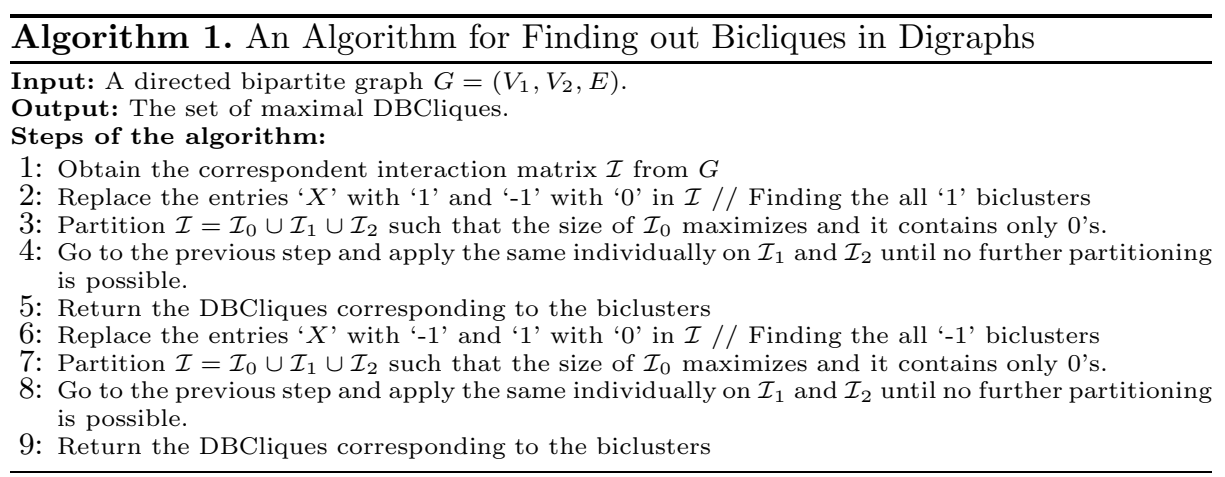

of interactions, viz., direct physical interactions and indirect interactions, categorized into 65 more specific types. The corresponding bipartite graph thus contains 19 vertices representing HIV-1 proteins and 1448 vertices representing human proteins, and 5134 edges representing the viral-host interactions. The interaction matrix of size $19 \times 1448$ corresponding to the bipartite graph is constructed. All the 65 interaction types are biologically classified into three types regulating, regulated by and two-way (where the regulation is bidirectional). As per this, an entry ' 0 ' in the matrix denotes the absence of interaction between the corresponding pair of HIV-1 and human proteins, an entry of ' 1 ' represents the regulation of the HIV-1 protein on the human protein, an entry of '-1' denotes that the HIV-1 protein is regulated by the human protein and ' $X$ ' represents a two-way interaction. Now, to find out the all ' 1 ' and all ' -1 ' biclusters from this, we applied the element-replacement strategies (steps 2 and 6) given in Algorithm 1 and the resulting binary matrices are processed further.

The available codes of Bimax biclustering method, as implemented in the BicAT toolbox 1, is employed to iteratively find out the matrix partitions of the binary data matrices. The lower bound of HIV-1 and human protein sizes are both set to 3 for obtaining a bicluster, i.e., we aim to find only those DBCliques which contain at least $3 \mathrm{HIV}-1$ proteins and 3 human proteins. With this restriction, the numbers of all ' 1 ' and all ' -1 ' DBCliques are found to be 113 and 25, respectively. Further neglecting don't care entries in these matrices, we ended up with 54 and 7 strong DBCliques, respectively for the all ' 1 ' and all '-1' cases. Several of these bicliques forms a strong (with high clustering co-efficient) bipartite subgraph which contains the HIV-1 proteins like env_gp120, pol_RT, tat, etc. On the other side, many of the human proteins like CD4, LCK, MAPK1 etc. and also several kinase proteins are obtained within this module. There are several literature evidences where CD4 has been reported as an important cell membrane protein that act as the receptor and co-receptor for the viral entry, respectively [2. The details of the DBCliques obtained are listed in Table 1 .

The all ' 1 ' and all ' -1 ' submatrices obtained are nothing but the representatives of strong modulated regulation between two sets of proteins. It becomes evident from the maximum sizes of the DBCliques that the immunodeficiency signal passing in either directions between HIV-1 and human proteins are equally 
Table 1. The DBCliques obtained from the HIV-1-human protein interaction network containing at least three HIV-1 and human proteins each. The size of a DBClique is defined based on the number of edges it contains.

\begin{tabular}{|c|c|c|c|}
\hline Bicluster type & Don't care allowed & \# DBCliques obtained & $\begin{array}{c}\text { Maximum size } \\
\text { (HIV-1, Human) }\end{array}$ \\
\hline \hline All '1' & Yes & 113 & $(6,5)$ \\
\hline All '-1' & Yes & 25 & $(3,13)$ \\
\hline All '1' & No & 54 & $(4,5)$ \\
\hline All '-1' & No & 7 & $(3,8)$ \\
\hline
\end{tabular}

significant. Further analysis from the Gene Ontology (GO) also shows the significant $p$-values $(<1.0 \mathrm{E}-3)$ for several of the DBCliques obtained representing highly coherent groups of regulatory functional proteins. As for example, consider the largest-sized and strong DBClique corresponding to the all '-1' bicluster. It consists of three HIV-1 proteins tat, env_gp120 and matrix, and eight heat shock $70 \mathrm{kDa}$ proteins (1A, 1B, 2, 4, 5, 6, 8 isoform 1 and 9 precursor) of human. These proteins are generally expressed when cells are exposed to elevated temperatures or other stresses likewise the entry of viral proteins. In fact, the env_gp120 protein is associated with the insertion of viral proteins in the host body in case of AIDS. Thus, this substructure is a biologically significant regulatory module in passing the immunodeficiency signal in human.

We have further carried out relative studies between the proposed method and several other algorithms to show the enhanced biological significance of the DBCliques in comparison with the conventional bicliques. All these algorithms are applied on the binary data matrix obtained from the interaction network ignoring directions. The comparative results are shown in Table 2 . Notably, every biclique is a DBClique but not the vice versa, and therefore DBCliques include additional information in their substructures. This is reflected from the better $p$-values obtained representing more biological coherence for DBCliques as compared to the bicliques. In fact, some of these results appear to be very insignificant producing poor $p$-values $(>1.0 \mathrm{E}-2)$. Thus, it appears that the DBCliques are significant substructures in a regulatory biological network.

Table 2. Comparison of the largest bicliques (consisting of at least three HIV-1 and human proteins) derived by various algorithms from the HIV-1-human protein interaction network. The proposed method exclude the Don't care conditions and returns DBCliques. Crossed cells in the third column represent insignificant $p$-values.

\begin{tabular}{|c|c|c|c|c|}
\hline Analytical details & Bimax & CC & ISA & Proposed \\
\hline \hline \# Bicliques obtained & 197 & 60 & 10 & 61 \\
\hline Largest biclique found & $(4,9)$ & $(19,392)$ & $(5,76)$ & $(3,8)$ \\
\hline Best $p$-value from GO & $1.9 \mathrm{E}-6$ & $\times$ & $\times$ & $2.3 \mathrm{E}-12$ \\
\hline $\begin{array}{c}\text { Best annotation } \\
\text { (GO Term) }\end{array}$ & $\begin{array}{c}\text { Regulation of cytokinesis } \\
\text { (GO:0032465) }\end{array}$ & $\begin{array}{c}\text { Not } \\
\text { applicable }\end{array}$ & $\begin{array}{c}\text { Not } \\
\text { applicable }\end{array}$ & $\begin{array}{c}\text { Response to protein } \\
\text { stimulus (GO:0051789) }\end{array}$ \\
\hline
\end{tabular}




\section{Concluding Remarks}

The problem of finding bicliques in directed bipartite graphs is addressed in this paper based on an optimal biclustering algorithm. We provide exact solutions to this problem and it is demonstrated that the problem reduces to a special class of biclustering problem. The analysis on the interaction network of HIV-1 and human proteins demonstrates the effectiveness of the proposed methodology and its usefulness.

\section{Acknowledgments}

A part of the work was carried out when S. Bandyopadhyay visited the Max Planck Institute for Informatics, Saarbrücken, Germany, and U. Maulik visited the German Cancer Research Center, Heidelberg, Germany, with Humboldt Fellowship for Experienced Researchers in 2010.

\section{References}

1. Barkow, S., Bleuler, S., Prelić, A., Zimmermann, P., Zitzler, E.: BicAT: a Biclustering Analysis Toolbox. Bioinformatics 22(10), 1282-1283 (2006)

2. Brass, A.L., Dykxhoorn, D.M., Benita, Y., Yan, N., Engelman, A., Xavier, R.J., Lieberman, J., Elledge, S.J.: Identification of Host Proteins Required for HIV Infection Through a Functional Genomic Screen. Science 319(5865), 921-926 (2008)

3. Cheng, Y., Church, G.: Biclustering of Expression Data. In: Proceedings of the 8th ISMB Conference, AAAI Press, pp. 93-103. AAAI Press, Menlo Park (2000)

4. Ding, C., Zhang, Y., Li, T.: Biclustering Protein Complex Interactions with a Biclique Finding Algorithm. In: Proceedings of the Sixth International Conference on Data Mining, Hong Kong, pp. 178-187 (2006)

5. Fu, W., Sanders-Beer, B.E., Katz, K.S., Maglott, D.R., Pruitt, K.D., Ptak, R.G.: Human immunodeficiency virus type 1 , human protein interaction database at NCBI. Nucleic Acids Research 37(Database Issue), D417-D422 (2009)

6. Madeira, S.C., Oliveira, A.L.: Biclustering algorithms for biological data analysis: a survey. IEEE/ACM Transactions on Computational Biology and Bioinformatics 1, 24-45 (2004)

7. Pandey, G., Atluri, G., Steinbach, M., Myers, C.L., Kumar, V.: An Association Analysis Approach to Biclustering. In: Proceedings of the 15th ACM SIGKDD Conference on Knowledge Discovery and Data Mining, Paris, France (2009)

8. Prelić, A., Bleuler, S., Zimmermann, P., Wille, A., Bühlmann, P., Gruissem, P., Hennig, L., Thiele, L., Zitzler, E.: A systematic comparison and evaluation of biclustering methods for gene expression data. Bioinformatics 22(9), 1122-1129 (2006)

9. Tanay, A., Sharan, R., Shamir, R.: Discovering statistically significant biclusters in gene expression data. Bioinformatics 18, S136-S144 (2002) 